\title{
ISOMETRIC MULTIPLIERS AND ISOMETRIC ISOMORPHISMS OF $l_{1}(S)$
}

\author{
CHARLES D. LAHR
}

\begin{abstract}
Let $S$ be a commutative semigroup and $\Omega(S)$ the multiplier semigroup of $S$. It is shown that $T$ is an isometric multiplier of $l_{1}(S)$ if and only if there exists an invertible element $\sigma \in \Omega(S)$ and a complex number $\lambda$ of unit modulus such that $T(\alpha)=\lambda \sum_{x \in S} \alpha(x) \delta_{\sigma(x)}$ for each $\alpha$ $=\sum_{x \in S} \alpha(x) \delta_{x} \in l_{1}(S)$.

Also, if $S_{1}$ and $S_{2}$ are commutative semigroups, and $L$ is an isometric isomorphism of $l_{1}\left(S_{1}\right)$ into $l_{1}\left(S_{2}\right)$, then it is proved that there exist a semicharacter $\chi,|\chi(x)|=1$ for all $x \in S_{1}$, and an isomorphism $i$ of $S_{1}$ onto $S_{2}$ such that $L(\alpha)=\sum \chi(x) \alpha(x) \delta_{i(x)}$ for each $\alpha=\sum_{x \in S_{1}} \alpha(x) \delta_{x} \in l_{1}\left(S_{1}\right)$.
\end{abstract}

1. Introduction. Let $S$ be a commutative semigroup. As usual, $l_{1}(S)$ denotes the Banach space of all complex functions $\alpha: S \rightarrow \mathrm{C}$ such that $\|\alpha\|$ $=\sum_{x \in S}|\alpha(x)|$ is finite. Letting $\delta_{x} \in l_{1}(S)$ represent the point mass at $x \in S$, an arbitrary element $\alpha$ of $l_{1}(S)$ is of the form $\alpha=\sum_{x \in S} \alpha(x) \delta_{x}, \alpha(x) \in \mathbf{C}$ for all $x \in S$; in fact, $\alpha(x) \neq 0$ for at most countably many elements of $S$. The linear space $l_{1}(S)$ becomes a commutative Banach algebra under the convolution product

$$
\alpha * \beta=\sum_{x \in S} \sum_{u, v ; u v=x} \alpha(u) \beta(v) \delta_{x},
$$

where $\alpha$ is as above and $\beta=\sum_{x \in S} \beta(x) \delta_{x} \in l_{1}(S)$. Further, $\hat{S}$ denotes the set of all semicharacters on $S$, that is, the set of all bounded nonzero functions $\chi: S \rightarrow \mathbf{C}$ such that $\chi(x y)=\chi(x) \chi(y)$ for all $x, y \in S$. For a fuller treatment of $l_{1}(S)$, consult [3].

Given a semigroup $S$, define $\Omega(S)$ to be the set of all functions $\sigma: S \rightarrow S$ having the property that $\sigma(x y)=x \sigma(y)$ for all $x, y \in S$. Under the operation of composition of functions, $\Omega(S)$ is a semigroup and is called the multiplier semigroup of $S$. Note that $\Omega(S)$ always has an identity $e$, the identity function on $S$. Throughout this paper assume that $\Omega(S)$ is commutative: a sufficient condition for the commutativity of $\Omega(S)$ is that $l_{1}(S)$ is semisimple. For weaker conditions implying commutativity and a more extensive discussion of $\Omega(S)$, consult [4, Proposition 4.1].

A bounded linear operator $T: l_{1}(S) \rightarrow l_{1}(S)$ is called a multiplier of

Received by the editors August 18, 1975.

AMS (MOS) subject classifications (1970). Primary 43A10, 43A20.

Key words and phrases. Isometric multiplier, $l_{1}$-algebra. 
$l_{1}(S)$ if $T(\alpha * \beta)=\alpha * T(\beta)$ for all $\alpha, \beta \in l_{1}(S)$. The set of multipliers of $l_{1}(S)$ is a commutative Banach algebra of operators under operator norm and is denoted $\Re\left(l_{1}(S)\right)$. An operator $T \in \mathscr{R}\left(l_{1}(S)\right)$ is an isometric multiplier of $l_{1}(S)$ if $T$ is a one-to-one mapping of $l_{1}(S)$ onto $l_{1}(S)$ with the property that $\|T(\alpha)\|=\|\alpha\|$ for all $\alpha \in l_{1}(S)$. For a general discussion of multipliers of Banach algebras and isometric multipliers, see [5].

Each element $\tau=\sum_{\sigma \in \Omega(S)} \tau(\sigma) \delta_{\sigma} \in l_{1}(\Omega(S))$ determines a multiplier $T_{\tau}$ of $l_{1}(S)$ as follows: defining $T_{\tau}$ first at each point mass $\delta_{x}$ of $l_{1}(S)$ by $T_{\tau}\left(\delta_{x}\right)$ $=\sum_{\sigma \in \Omega(S)} \tau(\sigma) \delta_{\sigma(x)}, x \in S$, extend $T_{\tau}$ linearly to the subspace $P$ of $l_{1}(S)$ spanned by the set of point masses; $T_{\tau}$ becomes a bounded operator on $l_{1}(S)$ by observing that $T_{\tau}$ is bounded on $P$ and that $P$ is dense in $l_{1}(S)$ [4, Proposition 4.2]. For each $\sigma \in \Omega(S), T_{\sigma}$ will denote the multiplier $T_{\delta_{\sigma}}$.

If $G$ is a locally compact group, denote by $L_{1}(G)$ the group (Banach) algebra of Haar integrable functions on $G$ under convolution multiplication. It is a well-known result that the isometric multipliers of $L_{1}(G)$ consist of scalar multiples of translation operators [7, Theorem 3]. The next section of this paper, $\S 2$, is devoted to a discussion of the isometric multipliers of $l_{1}(S)$. It is shown that if $T$ is an isometric multiplier of $l_{1}(S)$, then there exist $\sigma \in \Omega(S)$ and $\lambda \in \mathbf{C},|\lambda|=1$, such that $T=\lambda T_{\sigma}$.

Moreover, it is also known that an isometric isomorphism of two group algebras induces an isomorphism of the underlying groups [6]. In $\$ 3$, Theorem 3.1 , an analogous result is obtained for $l_{1}$-algebras.

\section{Isometric multipliers of $l_{1}(S)$.}

\section{Proposition 2.1. Let $\sigma \in \Omega(S)$. Then}

(a) $T_{\sigma}$ is an isometric multiplier of $l_{1}(S)$ if and only if $\sigma$ is one-to-one and onto, and

(b) $\sigma$ is one-to-one and onto if and only if $\sigma$ is invertible in $\Omega(S)$.

Proof. (a) Let $T_{\sigma}$ be an isometric multiplier. Then the one-to-oneness of $T_{\sigma}$ implies that $T_{\sigma}\left(\delta_{x}\right)=T_{\sigma}\left(\delta_{y}\right)$ if and only if $x=y, x, y \in S$; hence, $\sigma(x)$ $=\sigma(y)$ if and only if $x=y$, and thus $\sigma$ is one-to-one. Similarly, $\sigma$ is onto.

If, now, $\sigma$ is one-to-one and onto, then $T_{\sigma}\left(\delta_{x}\right)=\delta_{\sigma(x)}, x \in S$, shows that $T_{\sigma}$ is a one-to-one map of the set of point masses $\left\{\delta_{x}: x \in S\right\}$ onto itself. Hence, if $\alpha=\sum \alpha(x) \delta_{x} \in l_{1}(S)$, then $T_{\sigma}(\alpha)=\sum_{x \in S} \alpha(x) \delta_{\sigma(x)}$ is such that $\|\alpha\|$ $=\left\|T_{\sigma}(\alpha)\right\|$.

(b) If $\sigma$ is one-to-one and onto, then for $x \in S$, define $\sigma^{-1}(x)=y$ if and only if $\sigma(y)=x$. Then $\sigma^{-1}(x z)=y \Rightarrow x z=\sigma(y)$, and $\sigma^{-1}(z)=r \Rightarrow z$ $=\sigma(r)$, which implies that $\sigma(x r)=x \sigma(r)=\sigma(y)$ and hence $x r=y$ since $\sigma$ is one-to-one, or $\sigma^{-1}(x z)=y=x \sigma^{-1}(z)$; that is $\sigma^{-1} \in \Omega(S)$.

Conversely, if there exists $\sigma^{-1} \in \Omega(S)$ such that $\sigma \sigma^{-1}=e$, then $\sigma(x)$ $=\sigma(y)$ implies $x=y$ (showing $\sigma$ is one-to-one), and for a given $z \in S$, $\sigma\left(\sigma^{-1}(z)\right)=z$ (showing $\sigma$ is onto).

Define 


$$
\begin{aligned}
I & =\{\sigma \in \Omega(S): \sigma \text { is one-to-one and onto }\} \\
& =\{\sigma \in \Omega(S): \sigma \text { is invertible in } \Omega(S)\} .
\end{aligned}
$$

Observe that $I$ is never empty since $e \in I$. The next theorem shows that $I$ determines the set of isometric multipliers of $l_{1}(S)$.

THEOREM 2.1. Let $T \in \mathfrak{N}\left(l_{1}(S)\right)$. Then $T$ is an isometric multiplier of $l_{1}(S)$ if and only if $T=\lambda T_{\sigma}$ for some complex number $\lambda$ of unit modulus and some $\sigma \in I$.

Proof. Let $T$ be an isometric multiplier of $l_{1}(S)$. Then $T$ maps the unit ball of $l_{1}(S)$ onto the unit ball of $l_{1}(S)$, and, in particular, $T$ maps the extreme points of the unit ball onto the extreme points of the unit ball. Now, the collection of extreme points of the unit ball of $l_{1}(S)$ is the set $\left\{\lambda \delta_{x}: \lambda \in \mathbf{C},|\lambda|\right.$ $=1, x \in S\}\left[2\right.$, p. 81]. Hence, let us suppose that $T\left(\delta_{x}\right)=\lambda_{x^{\prime}} \delta_{x^{\prime}}, x \in S$. Then, for $x, y \in S$,

$$
\lambda_{(x y)^{\prime}} \delta_{(x y)^{\prime}}=T\left(\delta_{x y}\right)=\delta_{x} * T\left(\delta_{y}\right)=\delta_{x} * \lambda_{y^{\prime}} \delta_{y^{\prime}}=\lambda_{y^{\prime}} \delta_{x y^{\prime}}
$$

and also

$$
T\left(\delta_{x y}\right)=\delta_{y} * T\left(\delta_{x}\right)=\delta_{y} * \lambda_{x^{\prime}} \delta_{x^{\prime}}=\lambda_{x^{\prime}} \delta_{y x^{\prime}}
$$

Thus,

$$
(x y)^{\prime}=x y^{\prime}=x^{\prime} y \quad \text { and } \quad \lambda_{(x y)^{\prime}}=\lambda_{y^{\prime}}=\lambda_{x^{\prime}} \quad \text { for all } x, y \in S .
$$

Hence, there exists a unique complex number $\lambda$ of unit modulus such that $T\left(\delta_{x}\right)=\lambda \delta_{x^{\prime}}$ for all $x \in S$. Moreover, define a function $\sigma: S \rightarrow S$ by $\sigma(x)$ $=x^{\prime}, x \in S$. From above, the fact that $\sigma(x y)=x \sigma(y)=y \sigma(x)$ for all $x, y$ $\in S$ implies that $\sigma \in \Omega(S)$. Therefore, $T=\lambda T_{\sigma}, \sigma \in I$; by Proposition 2.1; and the implication is proved.

The converse follows immediately from Proposition 2.1 .

Part (b) of the following proposition shows that in many cases there may be very few isometric multipliers, indeed. Part (a) is an instance of Wendel's result for $L_{1}(G)$, where $G$ is a discrete group.

Proposition 2.2. (a) If $S$ has an identity $e$, then $I=\{x \in S: x$ is invertible in $S\}$. In particular, if $S$ is a group, $I=S$.

(b) If $S$ is an idempotent semigroup, then $I=\{e\}$ and the only isometric multiplier is the identity multiplier.

Proof. (a) If $S$ has an identity $e$, then $S=\Omega(S)$ and the result follows from Proposition 2.1(b).

(b) Let $\sigma \in \Omega(S), \sigma \neq e$; hence, there exist $x, y \in S$ such that $x$ $\neq y$ and $\sigma(x)=y$. Then $x y=x \sigma(x)=\sigma\left(x^{2}\right)=\sigma(x)=y$ implies that $y$ $=y^{2}=y \sigma(x)=\sigma(x y)=\sigma(y)$. Thus, $\sigma$ is not one-to-one and, therefore, is not in $I$. 
3. Isometric isomorphisms of $l_{1}(S)$. Let $S_{1}$ and $S_{2}$ be two commutative semigroups, and let $\Gamma=\left\{\chi \in \hat{S}_{1}:|\chi(x)|=1\right.$ for all $\left.x \in S_{1}\right\}$.

THEOREM 3.1. For each $\chi \in \Gamma$ and for each isomorphism $i: S_{1}$ $\rightarrow S_{2}$ of $S_{1}$ onto $S_{2}$, the linear operator $L: l_{1}\left(S_{1}\right) \rightarrow l_{1}\left(S_{2}\right)$, defined by $L(\alpha)$ $=\sum \chi(x) \alpha(x) \delta_{i(x)}$ for each $\alpha=\sum_{x \in S_{1}} \alpha(x) \delta_{x} \in l_{1}\left(S_{1}\right)$, is an isometric isomorphism of $l_{1}\left(S_{1}\right)$ onto $l_{1}\left(S_{2}\right)$. Conversely, if $L$ is an isometric isomorphism of $l_{1}\left(S_{1}\right)$ onto $l_{1}\left(S_{2}\right)$, then there exist $\chi \in \Gamma$ and an isomorphism $i$ of $S_{1}$ onto $S_{2}$ such that $L(\alpha)=\sum_{x \in S_{1}} \chi(x) \alpha(x) \delta_{i(x)}$ for each $\alpha=\sum_{x \in S_{1}} \alpha(x) \delta_{x} \in l_{1}\left(S_{1}\right)$.

Proof. Suppose $L$ is an isometric isomorphism of $l_{1}\left(S_{1}\right)$ onto $l_{1}\left(S_{2}\right)$. As in the proof of Theorem 2.1, $L$ maps the extreme points of the unit ball of $l_{1}\left(S_{1}\right)$ onto the extreme points of the unit ball of $l_{1}\left(S_{2}\right)$; say, $L\left(\delta_{x}\right)=\lambda_{x} \delta_{x^{\prime}}, x \in S_{1}$, $\lambda_{x} \in \mathbf{C},\left|\lambda_{x}\right|=1$. Then for $x, y \in S_{1}, \lambda_{x} \lambda_{y} \delta_{x^{\prime} y^{\prime}}=\lambda_{x} \delta_{x^{\prime}} * \lambda_{y} \delta_{y^{\prime}}=L\left(\delta_{x}\right)$ $* L\left(\delta_{y}\right)=L\left(\delta_{x y}\right)=\lambda_{x y} \delta_{(x y)^{\prime}}$ implies that $x^{\prime} y^{\prime}=(x y)^{\prime}$ and $\lambda_{x} \lambda_{y}=\lambda_{x y}$. Also note that $x, y \in S_{1}, x \neq y$, implies $x^{\prime} \neq y^{\prime}$ : for if $L\left(\delta_{x}\right)=\lambda_{x} \delta_{x^{\prime}}$, then for any $\lambda \in \mathbf{C},|\lambda|=1, L\left(\lambda \delta_{x} / \lambda_{x}\right)=\lambda \delta_{x^{\prime}}$; hence, the one-to-oneness of $L$ and the fact that $\lambda \delta_{x} / \lambda_{x} \neq \delta_{y}$ for any $\lambda \in \mathbf{C}$ imply that $L\left(\delta_{y}\right) \neq \lambda \delta_{x^{\prime}}$ for all $\lambda \in \mathbf{C}$, $|\lambda|=1$.

Define a map $i: S_{1} \rightarrow S_{2}$ by $i(x)=x^{\prime}, x \in S_{1}$; then $i$ is an isomorphism of $S_{1}$ onto $S_{2}$ since $x \neq y$ implies $x^{\prime} \neq y^{\prime}$ and $L$ maps the extreme points of $l_{1}\left(S_{1}\right)$ onto the extreme points of $l_{1}\left(S_{2}\right)$.

Finally, define a map $\chi: S_{1} \rightarrow \mathbf{C}$ by $\chi(x)=\lambda_{x}, x \in S_{1}$. Then the fact that $\chi(x y)=\lambda_{x y}=\lambda_{x} \lambda_{y}=\chi(x) \chi(y), x, y \in S_{1}$, and $|\chi(x)|=\left|\lambda_{x}\right|=1$ for all $x$ $\in S_{1}$ show that $\chi \in \Gamma$.

THEOREM 3.2. Let $L$ be an isometric isomorphism of $l_{1}\left(S_{1}\right)$ onto $l_{1}\left(S_{2}\right)$. Then

(a) $L$ induces an isomorphism $\bar{L}$ of $\Re\left(l_{1}\left(S_{1}\right)\right)$ onto $\Re\left(l_{1}\left(S_{2}\right)\right)$, and

(b) $\bar{L}$ maps the isometric multipliers of $l_{1}\left(S_{1}\right)$ onto the isometric multipliers of $l_{1}\left(S_{2}\right)$. Consequently, if $T_{\sigma}$ is an isometric multiplier of $l_{1}\left(S_{1}\right)$, then there exists an invertible multiplier $\sigma^{\prime} \in \Omega\left(S_{2}\right)$ and a complex number $\lambda_{\sigma}$ of unit modulus such that $L T_{\sigma} L^{-1}=\lambda_{\sigma} T_{\sigma^{\prime}}$.

Proof. (a) Define $\bar{L}$ : $\Re\left(l_{1}\left(S_{1}\right)\right) \rightarrow \Re\left(l_{1}\left(S_{2}\right)\right)$ by $\bar{L}(T)=L T L^{-1}, T$ $\in \mathscr{N}\left(l_{1}\left(S_{1}\right)\right)$. To see that $\bar{L}(T)$ actually belongs to $\Re\left(l_{1}\left(S_{2}\right)\right)$, observe that because $L, L^{-1}$ and $T$ are bounded linear operators, $L(T)$ is a bounded linear operator. Also, the boundedness and linearity of $\bar{L}(T)$ necessitate only verifying its multiplicative behavior on the set of point masses: for $x, y \in S_{2}$

$$
\begin{aligned}
\operatorname{LTL}^{-1}\left(\delta_{x y}\right) & =\operatorname{LT}\left(L^{-1}\left(\delta_{x}\right) * L^{-1}\left(\delta_{y}\right)\right) \\
& =L\left(L^{-1}\left(\delta_{x}\right) * T\left(L^{-1}\left(\delta_{y}\right)\right)\right) \\
& =\delta_{x} * L T L^{-1}\left(\delta_{y}\right)
\end{aligned}
$$

Thus, $\bar{L}(T) \in \Re\left(l_{1}\left(S_{2}\right)\right)$, as claimed. That $\bar{L}$ is one-to-one and onto is clear.

(b) From Theorem 2.1 and (a), it is sufficient to show that $L T_{\sigma} L^{-1}$ is an 
isometric multiplier of $l_{1}\left(S_{2}\right)$ for each isometric multiplier $T_{\sigma}$ of $l_{1}\left(S_{1}\right)$. Let $T_{\sigma}$ be an isometric multiplier of $l_{1}\left(S_{1}\right)$. Since $L$ maps point masses of $l_{1}\left(S_{1}\right)$ to scalar multiples of point masses of $l_{1}\left(S_{2}\right)$ in a one-to-one manner, and since $T_{\sigma}$ behaves similarly on $l_{1}\left(S_{1}\right)$, it is only necessary to compute the norm of $\bar{L}\left(T_{\sigma}\right)$ at an arbitrary point mass of $l_{1}\left(S_{2}\right)$. That is, if $x^{\prime} \in S_{2}$, it suffices to show that $\left\|L T_{\sigma} L^{-1}\left(\delta_{x^{\prime}}\right)\right\|=1$. However, observe that for a given $x^{\prime} \in S_{2}$ there exist $x \in S_{1}$ and $\lambda_{x} \in \mathbf{C},\left|\lambda_{x}\right|=1$, such that $L^{-1}\left(\delta_{x^{\prime}}\right)=\lambda_{x} \delta_{x}$. Then $T_{\sigma}\left(\lambda_{x} \delta_{x}\right)$ $=\lambda_{x} \delta_{\sigma(x)}$ implies that $L\left(\lambda_{x} \delta_{\sigma(x)}\right)=\lambda_{x}\left(\lambda_{x}\right)^{\prime} \delta_{\sigma(x)^{\prime}}$ for some $\left(\lambda_{x}\right)^{\prime} \in \mathbf{C},\left|\left(\lambda_{x}\right)^{\prime}\right|$ $=1, \sigma(x)^{\prime} \in \Omega\left(S_{2}\right)$; hence, $\left\|L T_{\sigma} L^{-1}\left(\delta_{x^{\prime}}\right)\right\|=\left|\lambda_{x}\left(\lambda_{x}\right)^{\prime}\right|=1$. Thus, the existences of $\lambda_{\sigma} \in \mathbf{C}$ and $T_{\sigma^{\prime}}$ defined in the statement of the theorem follow from Theorem 2.1.

It should be noted that there is a more general statement of Theorem 3.2(a) in [1, Theorem 1].

Although an isometric isomorphism of $l_{1}$-algebras can be extended to an isomorphism of the respective multiplier algebras, it is not true that an isomorphism of multiplier algebras induces an isomorphism of the underlying $l_{1}$-algebras. Let $S_{1}$ be the set of negative integers under the operation of maximum multiplication; $\Omega\left(S_{1}\right)=S_{1} \cup\{e\}$; that is, $\Omega\left(S_{1}\right)$ is obtained from $S_{1}$ by adjoining an identity. Let $S_{2}=S_{1} \cup\{e\}$; clearly, $\Omega\left(S_{2}\right)=S_{2}$. $\Re\left(l_{1}\left(S_{1}\right)\right)$ $=l_{1}\left(\Omega\left(S_{1}\right)\right)$ since $l_{1}\left(S_{1}\right)$ has a bounded approximate identity [4], and $\Re\left(l_{1}\left(S_{2}\right)\right)=l_{1}\left(S_{2}\right)$. Certainly, $\pi\left(l_{1}\left(S_{1}\right)\right)$ is isomorphic (isometrically) to $\Re\left(l_{1}\left(S_{2}\right)\right)$, but $l_{1}\left(S_{1}\right)$ is not isomorphic to $l_{1}\left(S_{2}\right)$.

\section{REFERENCES}

1. F. T. Birtel, Isomorphisms and isometric multipliers, Proc. Amer. Math. Soc. 13 (1962), 204-210. MR 31 \#620.

2. M. M. Day, Normed linear spaces, Springer-Verlag, New York, 1962. MR 26 \#2847.

3. E. Hewitt and H. S. Zuckerman, The $l_{1}$-algebra of a commutative semigroup, Trans. Amer. Math. Soc. 83 (1956), 70-97. MR 18, 465.

4. C. D. Lahr, Multipliers for certain convolution measure algebras, Trans. Amer. Math. Soc. 185 (1973), 165-181. MR 48 \# 11912.

5. R. Larsen, The multiplier problem, Springer-Verlag, Berlin and New York, 1969.

6. J. G. Wendel, On isometric isomorphism of group algebras, Pacific J. Math. 1 (1951), 305-311. MR 14, 246.

7. Left centralizers and isomorphisms of group algebras, Pacific J. Math. 2 (1952), 251-261. MR 14, 246.

Department of Mathematics, Dartmouth College, Hanover, New Hampshire 03755 